\title{
A view from the ethics committee
}

W hen I stepped down as the dean of Department of Medicine, University of Manitoba Medical School, I thought that with my extra time I could amuse myself and contribute by accepting one of the least well rewarded but most important university jobs - serving on the research ethics committee. I volunteered and was accepted instantly. After a year in the trenches, the chairmanship of one of our committees became vacant, and I was given the job, presumably because I know how to run a meeting. It has been a very enlightening experience, and I would like to share my impressions of the current state of the research ethics enterprise. Although my experience is limited to my own institution, I am sure that most of the problems that I will cite are common.

Research ethics is a growth industry, for a number of reasons. First, there have been some highly publicized research disasters, involving both fraud and injury of experimental subjects. Second, the volume of clinical research has increased enormously. Finally, the renewed interest in clinical ethics has spurred collateral interest in research ethics, although the two are really different subjects. There may be other reasons, but whatever they are, the effect is to confront research ethics committees with huge burdens.

The typical research ethics committee is staffed by volunteers. Though the chair may receive an honorarium, the remainder of the committee serves entirely because they wish to do so. Members include students, faculty and interested lay people, all of whom, with no tangible reward, shoulder a substantial workload. The committee that I chair reviews well over 200 new protocols/year at 11 meetings. It is not uncommon to go over 20 of them in an afternoon, presenting the experimental plan, trying to judge its merit and reviewing the informed consent in detail. I normally spend 8 to $12 \mathrm{~h}$ preparing for a meeting, and I go over protocols in less detail than the committee members who have been assigned to them. For faculty members, who constitute the core of the committee by virtue of their numbers and expertise, service on the committee is expensive and time consuming, and is not attended by a great deal of recognition on the part of department heads or colleagues. Finding interested faculty and convincing them to accept an appointment on the committee demands a more or less continuous effort. Those who do accept and serve ought to be recognized as heroes by the rest of us.

\section{A major problem} encountered by our committee is evaluating the merits of the proposed research. In the old days, it was simple in that most clinical research had been peer reviewed by its funders. Now, pharmaceutical companies sponsor the vast majority of clinical research. They have a variety of objectives, and they do not subject their

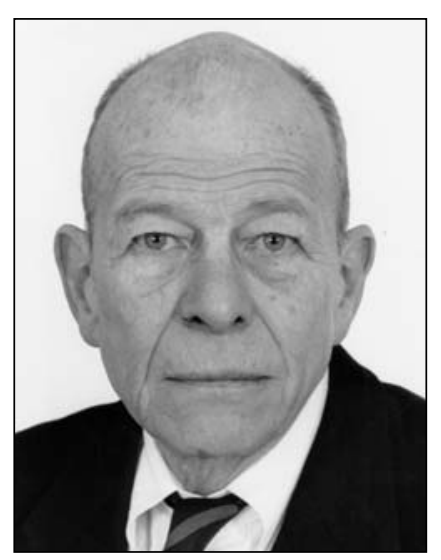

Nick R Anthonisen proposals to independent peer review. Committees are told and believe that bad science is unethical, and are, therefore, in the position of having to judge the quality of the science in some way. While the committee has some scientific expertise, it cannot cover all fields of research, and not uncommonly, is forced to seek a separate outside opinion. Needless to say, this may be a hit or miss proposition, which does not sit well with the investigator. In these matters, scientific quality interacts with the risks of the proposal, in that relatively trivial phase 4 trials may be approved if the risks to the participants are also trivial. There have been suggestions that there be some national review process for multicentre clinical trials by pharmaceutical companies, and this seems to be a good idea.

A second problem is the institutionalization or perhaps 'lawyerization' of the informed consent. A rule of thumb is that the informed consent document should be comprehensible by someone with a grade 8 education. I think that this is a rare event, and it is neither the investigator's nor the committee's fault. So much has to be conveyed to the participant that the document is very frequently 10 pages long, and crammed with descriptions of procedures, unwanted side effects, issues regarding confidentiality, etc. Because all the bases have to be covered, the description of the study and its ramifications is so long and detailed that people with substantially more than a grade 8 education find it difficult to understand. One wonders how truly informed most consents are, and I suspect that participants join studies largely because they trust the investigative team to do the right thing. This is probably what occurred before the advent of 
detailed informed consent, so the document may have more legal than ethical significance. There appears to be no way out of this dilemma.

Finally, I must comment on the state of clinical research in general as viewed from the vantage point of the ethics committee. We are told that there is more clinical research going on than ever before, and that enormous sums of money are being poured into these efforts. This is certainly true, but whether there is a net benefit to academic clinical research is less clear, at least to me. As noted above, pharmaceutical companies fund the vast majority of current clinical research on a contractual basis. It is not driven by investigator curiosity, to put it mildly. Indeed, most investigators have little or nothing to do with developing the pro- tocol, which is to my mind the most important aspect of any research. It follows that this kind of research is nearly useless for training purposes, and is probably not helpful to serious investigators in terms of career advancement. There is, however, one bright spot on the horizon. My committee has recently reviewed an increasing number of clinical trials sponsored by the Canadian Institutes for Health Research; these are invariably well designed and ask important questions. Needless to say, they have also been peer reviewed.

Nick R Anthonisen MD Editor-in-Chief Canadian Respiratory Journal 


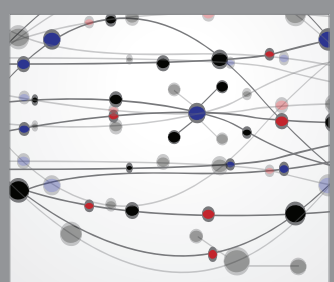

The Scientific World Journal
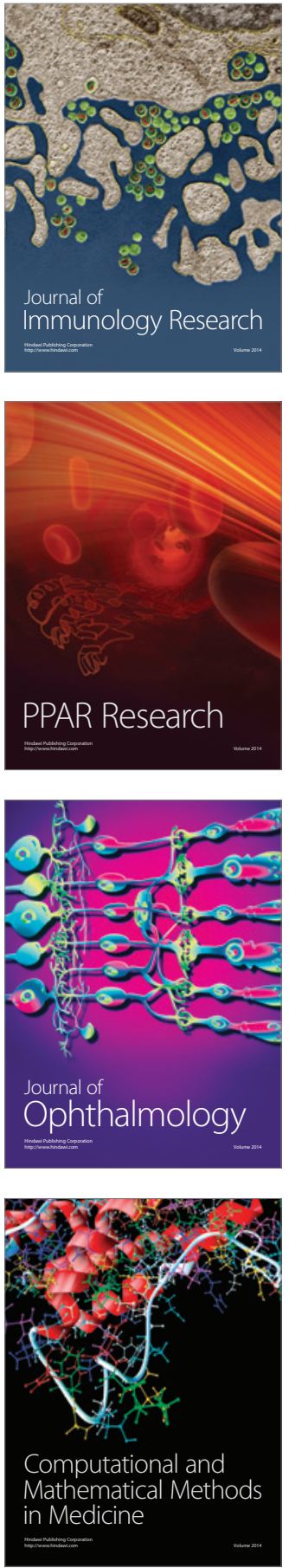

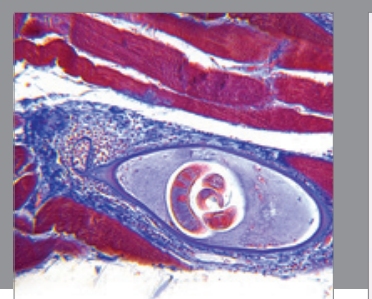

Gastroenterology Research and Practice

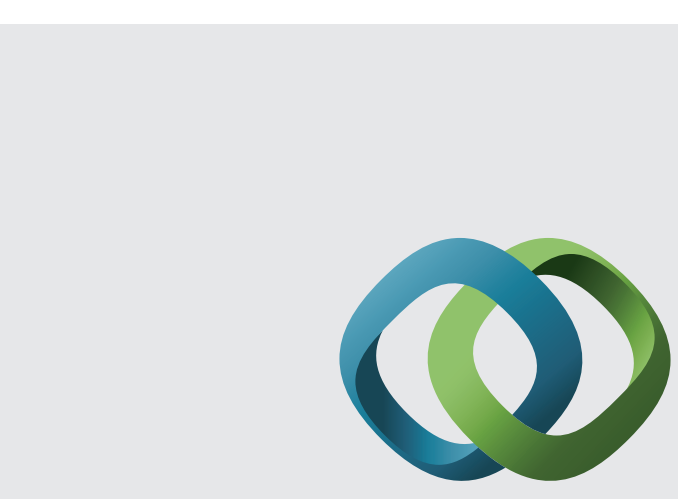

\section{Hindawi}

Submit your manuscripts at

http://www.hindawi.com
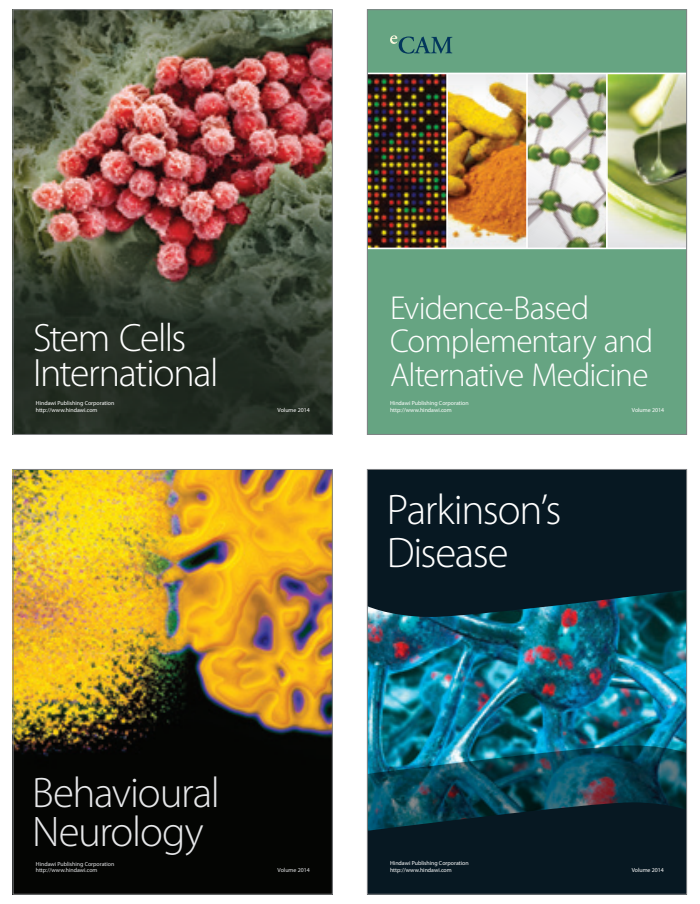
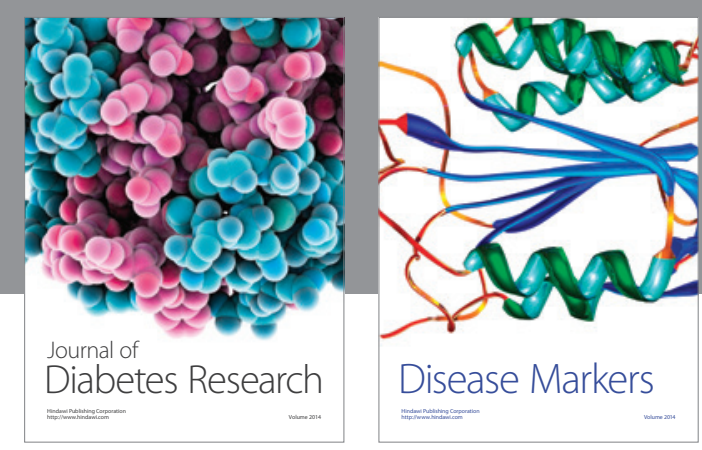

Disease Markers
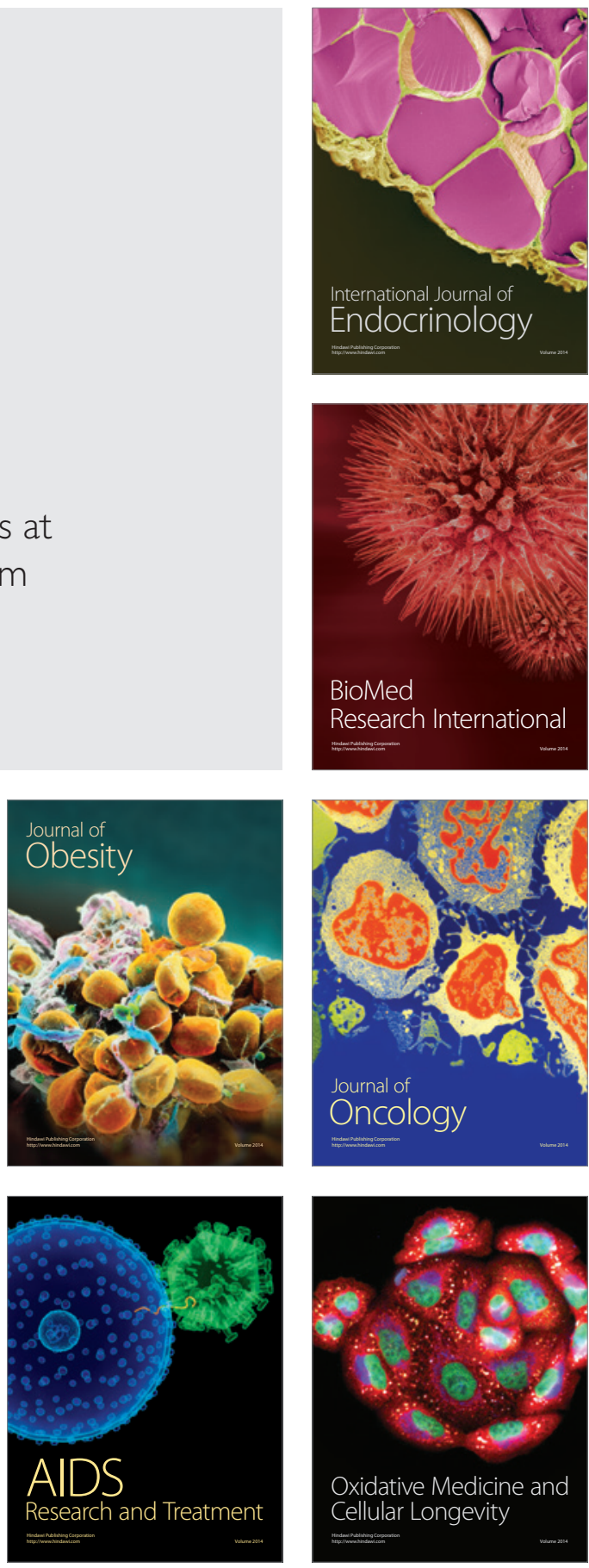